\title{
Comparing the effects of current circumcision techniques on dorsal root ganglia: an experimental study
}

\author{
Ozgur Caglar ${ }^{*}$, Remzi Arslan², Binali Firinci ${ }^{1}$, Muhammed Enes Aydin ${ }^{3}$, Erdem Karadeniz $^{4}$, Kemal Alp Nalci $^{5}$,
} Ahmet Bedii Salman ${ }^{1}$ and Mehmet Dumlu Aydin ${ }^{6}$

\begin{abstract}
Background: Circumcision is a surgical removal process of the preputium. Surgical excision and electric current cause neurodegeneration in the excised tissue-related neural network. This study investigates if circumcision and current surgical methods would cause histopathological changes in the penile tissue innervating pudendal nerve dorsal root ganglia.

Results: Vascular congestion occluded the microvessels, and degenerative changes were detected in postexcised penile tissue. Neuronal angulation, cytoplasmic condensation, nuclear shrinkage, and condensed nuclei were observed in the pudendal nerve ganglia. Moreover, the degenerated neuron (DN) densities of the pudendal nerve ganglia were $5 \pm 2,98 \pm 22,1093 \pm 185,236 \pm 43$ and $151 \pm 19$ in the control, the surgical circumcision, the monopolar cautery, the bipolar cautery and the thermocautery groups, respectively.

Conclusions: Although only the pudendal nerve endings are affected secondary to prepusium excision another pudendal axons can be damaged during bleeding control with cautery, the destruction of the neuron that sends this axon initiates a general inflammation in that ganglion. As a result, there may be a somatosensitive innervation defect in extrapenile tissues innervated by this ganglion. High-voltage electric devices should not be used unless obligatory.
\end{abstract}

Keywords: Circumcision, Electrocautery, Pudendal nerve, Dorsal root ganglia, Neurodegeneration

\section{Background}

Circumcision is one of the most common surgeries carried out in male children to treat phimosis or for cultural and religious reasons [1]. The penile artery, which is the main artery of the penis in the rabbit, is a branch of the internal pudendal artery. It divides into deep and dorsal penile arteries and creates an ischial arc and provides blood circulation to the penis and prepuceum [2]. Blood circulation of the penis is similar in humans. Various nerves of the pelvic plexus are responsible for autonomic control of pelvic visceral tissues. The major

\footnotetext{
* Correspondence: drozgurcaglar@yahoo.com

'Department of Pediatric Surgery, Medical Faculty, Ataturk University,

Erzurum, Turkey

Full list of author information is available at the end of the article
}

source of neurons of main penile nerves which innervate the corpus spongiosum and bulbourethral gland contains parasympathetic neurons in the Onuf's nucleus located in the parasympathetic region of the spinal cord which project to the penile crura and sympathetic nerves arising from sympathetic region of the spinal cord from L1 to L2 levels provide preganglionic innervation to ganglion cells in the main pelvic nerves. Somatic and autonomic fibres, including pelvic, hypogastric and commonly pudendal nerves, innervate the penile tissues [3]. The pudendal nerve is the primary coordinator of orgasmic sensation [4]. During circumcision surgery, the dissected nerves, arteries and veins are mostly pudendal nerves and dorsal penile arteries. Several circumcision techniques have been used; however, there has been 
considerable debate over which is the most usable and least complicated one in terms of bleeding [5], hazardous electrical current effects [6], thermocoagulation and the clamping method [7]. To elucidate the safest circumcision technique, we conducted an animal study using all current circumcision techniques and compared their neurodegenerative effects on dorsal root ganglia (DRG).

This study aims to investigate the neurovascular damage caused by the electric devices used for the circumcision procedure.

\section{Methods}

Experiments were carried out according to the guidelines set by the ethical committee of our faculty (ethical number, 17.09.2018/10). Rabbits were obtained from the laboratory animal centre (company name Aykut Bolu Deneysel Hayvan Üretim ve Tedarik Merkezi/Bolu/ Turkey). The rabbit model was chosen because it has preputium and the penis structure is similar to that of humans. Thirty-four male, 6-8-month-old New Zealand rabbits were divided into five groups as follows: five in the control (GI, $n=5$ ), five in the surgical circumcision (GII, $n=5$ ), eight in the monopolar cautery (GIII, $n=$ $8)$, eight in the bipolar cautery (GIV, $n=8)$ and eight in the thermocautery (GV, $n=8$ ) groups. All surgical procedures were performed under general anaesthesia. The authors other than the pathologist were aware of the group allocation of the experiment. Normal penile and pudendal network data were obtained for five normal animals. The study group was anaesthetised via the subcutaneous injection of a mixture of ketamine hydrochloride $(25 \mathrm{mg} / \mathrm{kg})$, lidocaine hydrochloride $(15 \mathrm{mg} / \mathrm{kg})$ and acepromazine $(1 \mathrm{mg} / \mathrm{kg})$. Circumcision was performed with surgical scissors, monopolar cautery $(20 \mathrm{~W} /$ $400 \mathrm{kHz}$; Petkot 600, Petaş) and thermocautery $\left(225^{\circ} \mathrm{C}\right.$, Warme Medizinische Qx-2100), following surgical region sterilisation with local antiseptics. Meanwhile, bipolar $(20 \mathrm{~W} / 400 \mathrm{kHz}$; Petkot 600, Petaş) and monopolar cautery were used for bleeding control (Fig. 1).

Electrocauterisation may have hazardous effects on motor, sensory, and autonomic nerves because living tissues can transmit electric currents to neural tissues and neural tissue damage occur due to electric energy is converted to thermal energy or heat [8]. Electrical injuries to nerves can be especially devastating. The damage depends on the duration of the electric current, the orientation and location of the cells in the current path, tissue resistance, tissue susceptibility and type, density, duration and the strength of the electric current. The mechanism of electrical injury can be thermal damage, vascular impairment, and histological or electrophysiological changes in peripheral nerves, or direct mechanical trauma [9]. Monopolar coagulation is more injurious than bipolar electrocoagulation [10].
The animals were kept for 3 weeks after surgery in personal cages in a standard laboratory room without medical treatment. Then, all animals were sacrificed, and their penile tissues together with their neurovascular compartments extending to the dorsal root ganglia of the pudendal nerve roots (NR) at the sacral level 3 were extracted, fixed in a $10 \%$ formalin solution for 1 week, for light microscopic analysis and then stained with haematoxylin and eosin. Post-circumcision views and dissection are shown in Fig. 2. The degenerated neuron (DN) densities of DRG were examined by the current histopathological methods. Cytoplasmic condensation, nuclear shrinking, cellular angulations and pericytoplasmic halo formation were used to identify neuronal degeneration. The physical dissector method was employed to distinguish living and DNs in pudendal nerve DRG, as shown in Fig. 3a, b. Statistical analysis was conducted to compare the DN densities in each group using the one-way ANOVA test in SPSS 20.0 for Windows $(p<0.005)$.

\section{Results}

\section{Anatomical findings}

All animals were healthy upon follow-up, and only one animal died during surgery. Neither autonomic nor somatosensitive findings were observed. Upon macroscopic examination, wound healing was nearly uncomplicated except for one phimosis case. Phimosis in using monopolar cautery was thought to be due to cautery damage and excessive preputial tissue left. Anatomical observations with magnifying devices revealed that all cases had some problems in the postcircumcision period. Oedematous penile-peripenile tissues, congested vessels and neuromatous masses of the cut nerves were noticed. Traumatic neuromatous masses are described as benign lesion that arises at site of prior trauma, surgery, amputation and named as scar, amputation or pseudoneuroma which develops as aberrant neural regeneration following injury. No elaboration and statistical study between groups was conducted on these findings.

\section{Histopathological results}

The generalised histopathological findings included burned fibrous and neurovascular bundles, vascular congestion and occluded microvessels detected in the postamputated parts of the glandular tissues and the periphery of the distal urethra. Neuronal angulation, cytoplasmic condensation, nuclear shrinkage and dense nuclei were detected in the pudendal nerve ganglia, meeting the neuronal degeneration criteria. The number of live neurons was higher in surgical circumcision than in bipolar cautery, thermocautery and monopolar cautery. DN numbers were found as $1093 \pm 185$ in 


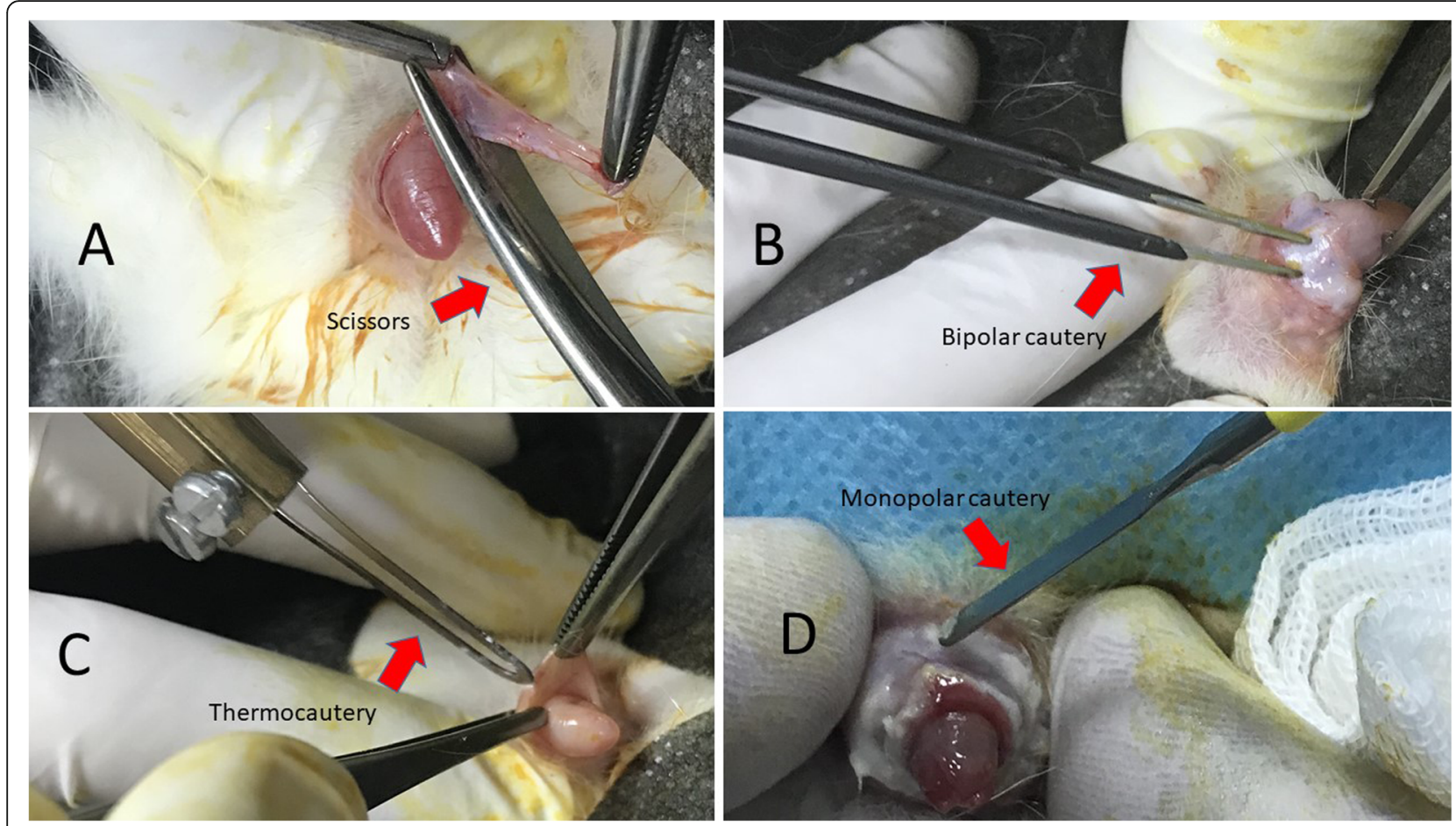

Fig. 1 Views of circumcision technique and cautery devices. a surgical circumcision, b bipolar cautery, c thermocautery, $\mathbf{d}$ monopolar cautery

monopolar cautery, $236 \pm 43$ in bipolar cautery, $151 \pm$ 19 in thermocoutery and $98 \pm 22$ in surgical circumcision, from the highest to the lowest (Table 1). Microscopical examination revealed that tissue adhesion, peridural fibrosis and scar formation at the operation site were more prominent in the monopolar cautery group. The migration of mononuclear cells and inflammation was observed abundantly in the monopolar cautery and thermocautery groups; however, it was slight or absent in the bipolar cautery and surgical circumcision groups.

The histopathological results were as follows: Figure 4 shows the penis, urethral mea and preputium in a normal rabbit; the penis and preputial tissue 3 weeks after circumcision; the dissected view of the partially healing oedematous penile-peripenile soft tissues; and dorsal

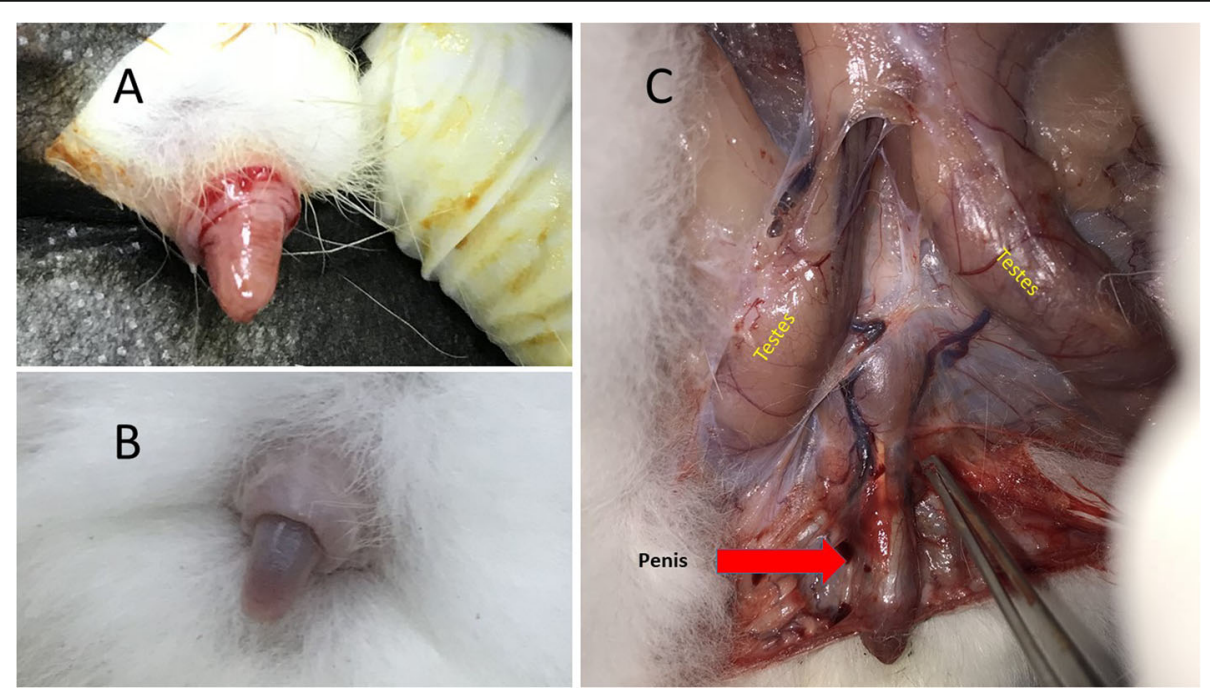

Fig. 2 Views of the penis after circumcision (a early postoperative, b late postoperative) and during dissection (c) 


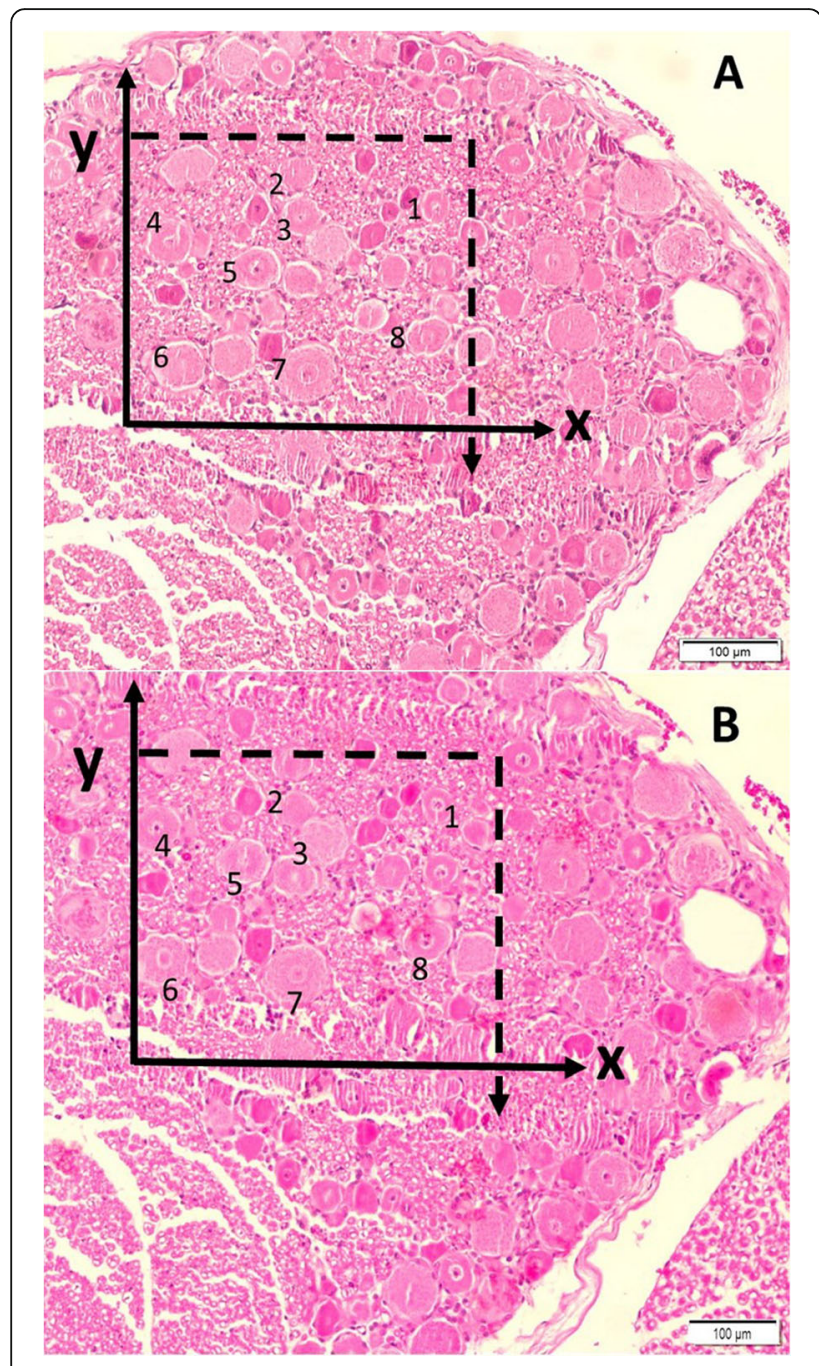

Fig. 3 Application of the physical dissector method, in which micrographs in the same fields of view $(\mathbf{a}, \mathbf{b})$ are taken from two parallel, adjacent thin sections separated by a distance of $5 \mathrm{~mm}$. Upper and right lines of unbiased counting frames represent the inclusion lines; the lower and left lines, including the extensions, are exclusion lines. Any neuron nucleolus reaching the inclusion lines was excluded, and nucleolus profiles hitting the inclusion lines and located inside the frame were counted as dissector particles unless their profiles were extended up to the lookup section. The number of neurons from the two dissectors occurs in a volume given by the product of the counting frame area and the distance between the sections. The numerical density of the neurons is calculated as NvGN $=\Sigma Q^{-} \mathrm{GN} / \mathrm{txA}$. In this application, the nucleoli marked with "1, 3, 6, 8" are dissector particles in $\mathbf{a}$, which disappeared in $\mathbf{b}$. The nucleoli marked "2, 4, 5, 7" are not dissector particles in $\mathbf{a}$, which disappeared in $\mathbf{b}(H \& E, 400, L M)$

penile neurovascular structures of a circumcised rabbit under anaesthesia just before scarification. Figure 5 displays the anatomical representation of the sacral spinal cord (SC), pudendal NR and dorsal root ganglions; spinal cord section at Onuf's nucleus level and the histopathological appearance of the spinal cord, the artery of
Table 1 Numerical results of study

\begin{tabular}{llllll}
\hline & Group I & Group II & Group III & Group IV & Group V \\
\hline $\mathrm{DND} /\left(\mathrm{n} / \mathrm{mm}^{3}\right)$ & $5 \pm 2$ & $98 \pm 22$ & $1093 \pm 185$ & $236 \pm 43$ & $151 \pm 19$
\end{tabular}

The values represent the mean \pm standard deviation, group I to $\mathrm{V}$ $D N D$ degenerated neuron density of pudendal ganglia

$p<0.005 \mathrm{Gl}$ and Gll one-way ANOVA

$p<0.00005 \mathrm{Gll}$ and GIII one-way ANOVA

$p<0.000001 \mathrm{Gl}$ and GIII one-way ANOVA

$p<0.0001 \mathrm{Gl}$ and GIV one-way ANOVA

$p<0.0005 \mathrm{Gl}$ and GV one-way ANOVA

$p<0.0005$ GIII and GV one-way ANOVA

$p<0.0005$ GIII and GIV one-way ANOVA

Adamkiewicz (AKA); and dorsal root ganglion neurons, nerve axons and spinal cord. The application of the physical dissector method is summarised in Fig. 3's legend. The histopathological appearance of DRG with normal and DNs and nerve fibres of an animal that underwent surgical circumcision is demonstrated in Fig. 6. The histopathological appearances of normal and DNs, including DRG, in animals that underwent the thermocautery and monopolar cautery procedures, are shown in Figs. 7 and 8. The histopathological appearance of NR and normal and DNs, including DRG, in an animal where bipolar cautery was conducted is shown in Fig. 9. Moreover, neurodegenerative changes in the neighbouring ganglia/neurons of affected ganglia were detected in the study group.

\section{Numerical results}

The mean total neuron count of the pudendal nerve ganglia was $21.950 \pm 1.870$ for the control animals. Degenerated neuron densities per cubic millimetre were as follows: $5 \pm 2,98 \pm 22,1093 \pm 185,236 \pm 43$ and 151 \pm 19 in the control, surgical circumcision, monopolar cautery, bipolar cautery and thermocautery groups. A summary of the numerical and statistical results is given in Table 1.

\section{Statistical results}

The statistical results were not significantly different between the control and surgical circumcision groups.

\section{Discussion}

Although circumcision has been recommended for religious, social and legal reasons, there is a hypothesis that the use of electric devices may be hazardous for the neurovascular compartment of the genital organs, such as the peripheral NR complex $[11,12]$. In our research, we aimed to investigate if preputium cutting in circumcision using modern surgical methods plays an important role in preputial excision injuries. Aydin et al. have reported that genital organs innervating pudendal nerve/taste rosea circuitry injuries may be responsible for disorgasmia causing by disruption of the erectile/ejaculatory/ somatosensitive feedback networks $[11,12]$. Circumcised 


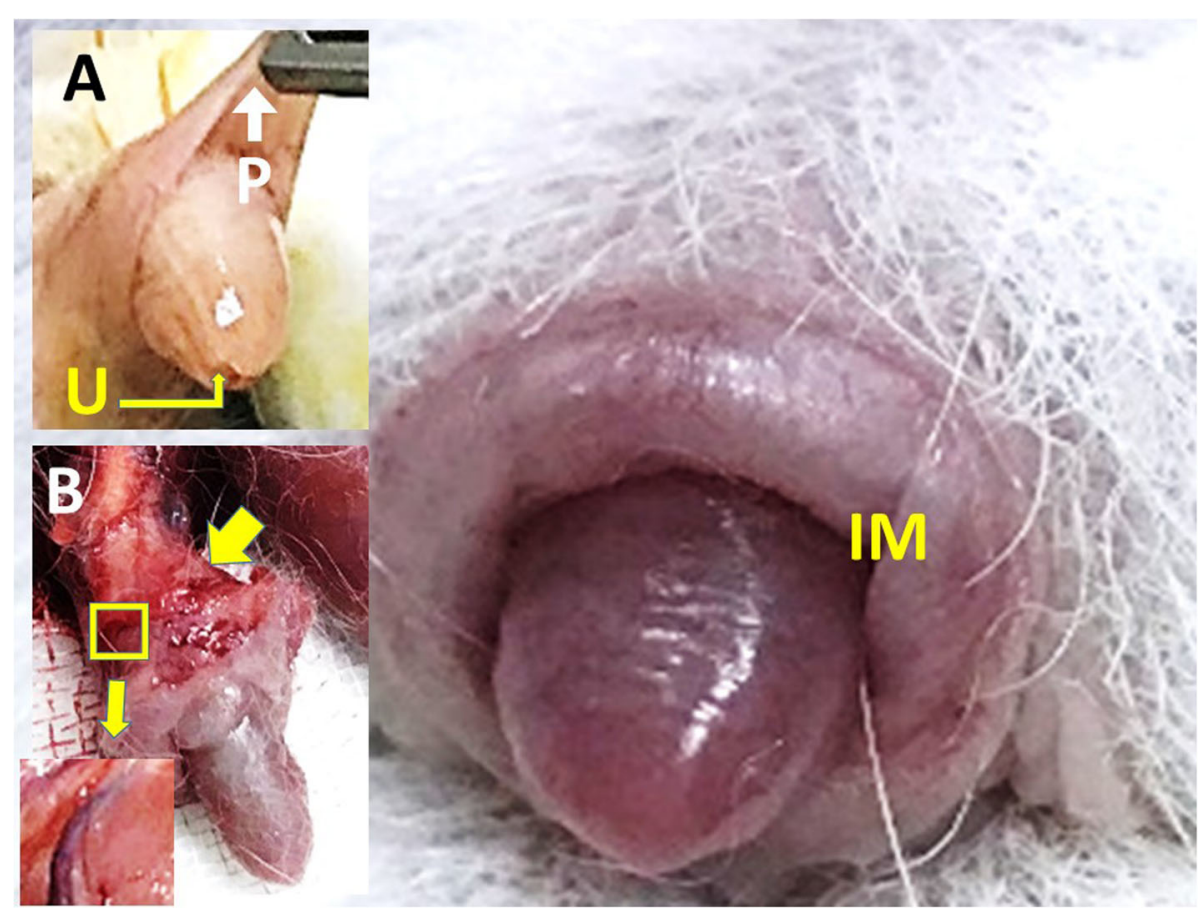

Fig. 4 a Appearance of the penis, urethral mea $(U)$ and preputium $(P)$ in a normal rabbit; the penis and preputial tissue (IM) 3 weeks after circumcision (base). $\mathbf{b}$ The dissected view of the partially healing oedematous penile-peripenile soft tissues (yellow arrow) and dorsal penile neurovascular structures (yellow square) of a circumcised rabbit under anaesthesia just before sacrification

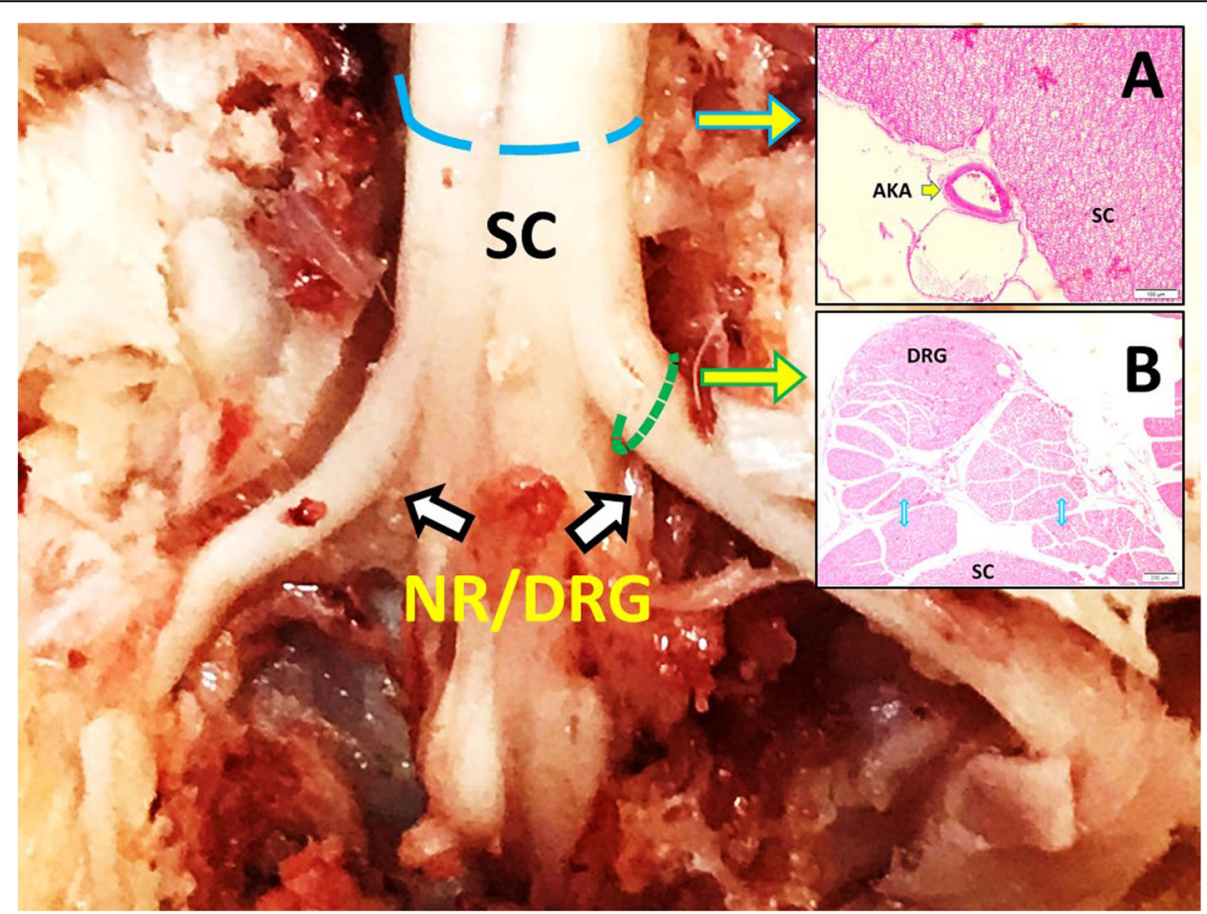

Fig. 5 Anatomical representation of sacral spinal cord (SC), pudendal nerve roots (NR) and dorsal root ganglions (DRG) (base); spinal cord section at Onuf's nucleus level (blue circle) and the histopathological appearance of the spinal cord (SC), the artery of Adamkiewicz (AKA) (LM, H\&E, $\times 10$ / A); and dorsal root ganglion neurons (DRG), nerve axons (blue arrows) and spinal cord (SC) (LM, H\&E, $\times 4 / B)$. 


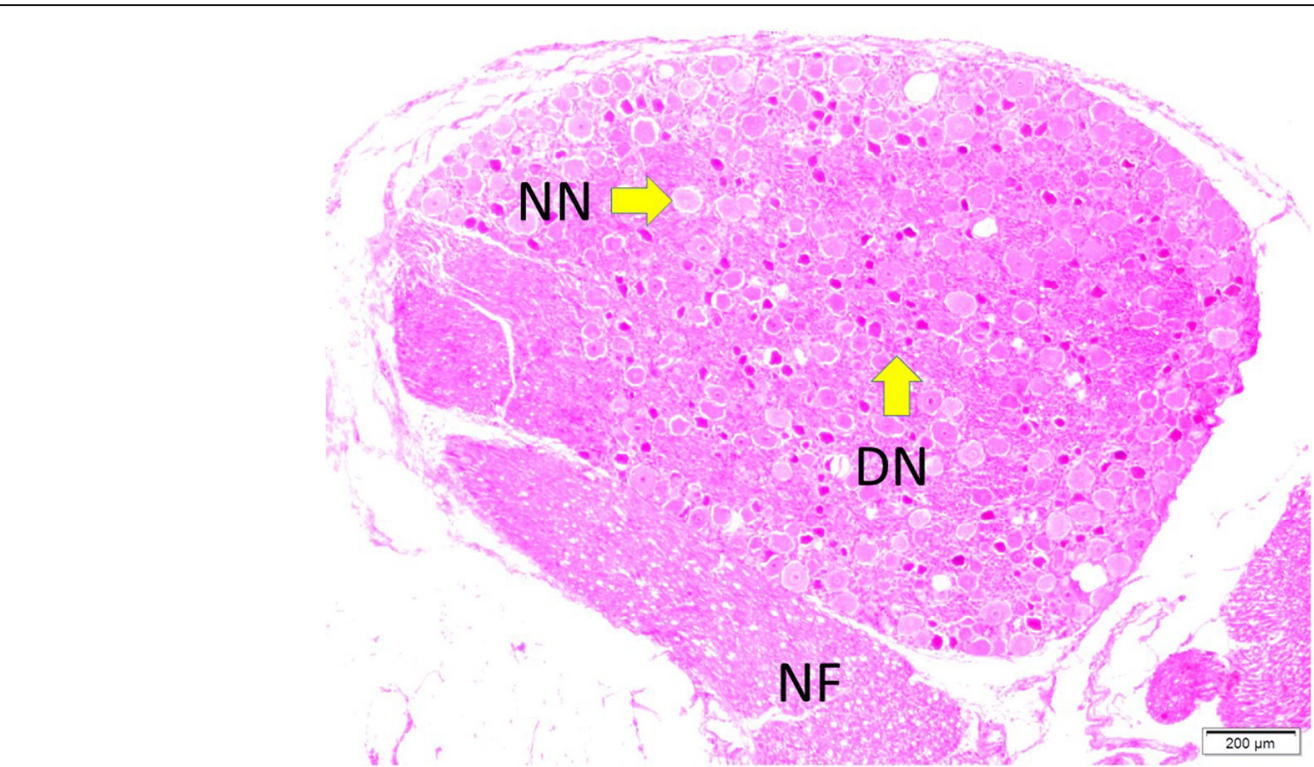

Fig. 6 Histopathological appearance of DRG with normal (NN) and degenerated (DN) neurons and nerve fibres (NF) are seen in an animal that underwent surgical circumcision

patients may suffer from some neurourological or psychosexual disorders because pudendal nerve network injuries could result in disorgasmia as reported by Aydin et al. [4]. Circumcision is used to treat phimosis and recurrent balanitis and reduce the risk of urinary tract infection in boys less than 1 year old with concomitant urinary tract abnormalities and is performed for religious, cultural and societal preferences.
Pudendal, pelvic and hypogastric nerves consist of somatic and autonomic fibres that innervate penile tissues and produce orgasmic sensations [3].

Understanding the anatomy of the penile neurovascular network in rabbits is necessary. Thus, we examined the branching patterns of their penile vessels. The internal pudendal artery and ischiatic arch are the primary sources, which are divided into two branches, that is the

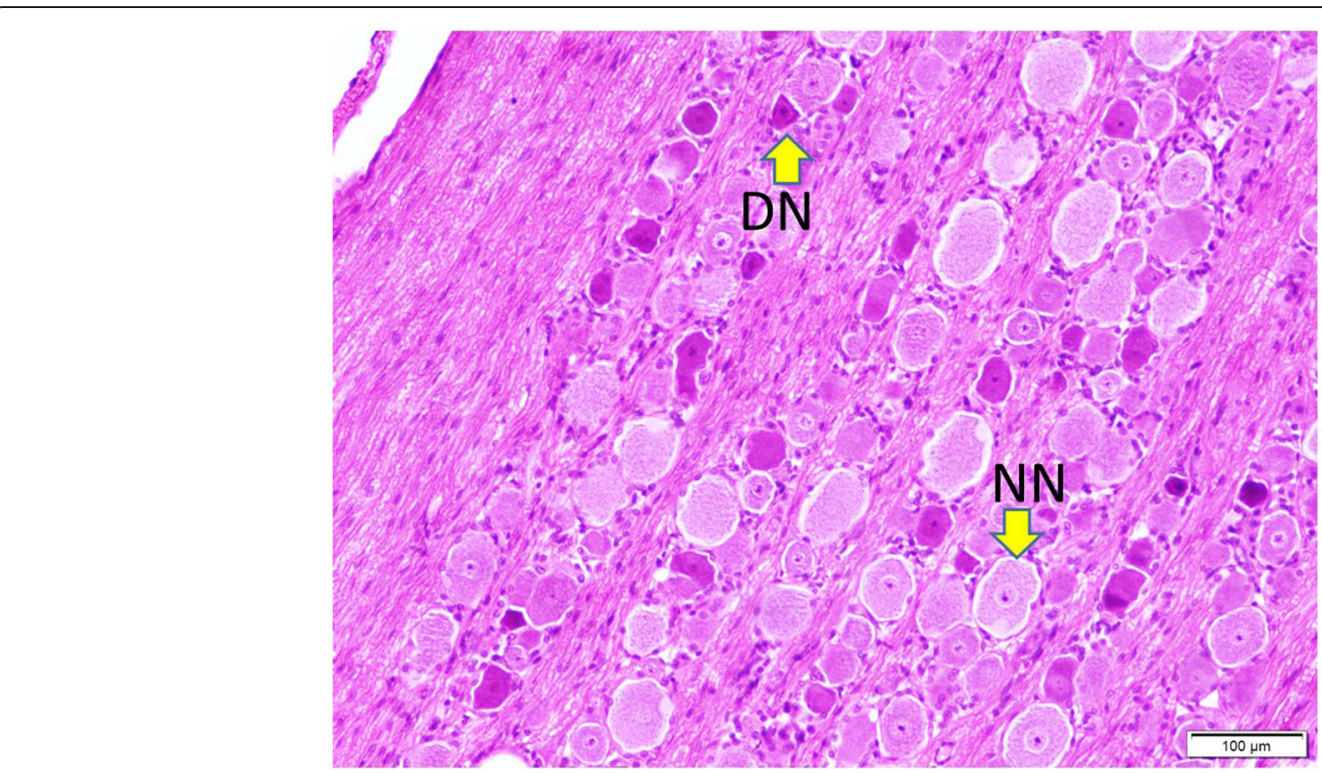

Fig. 7 Histopathological appearance of normal (NN) and degenerated (DN) neurons, including DRG, in an animal that underwent thermocautery $(\mathrm{LM}, \mathrm{H} \& \mathrm{E}, \times 10 / \mathrm{A})$ 


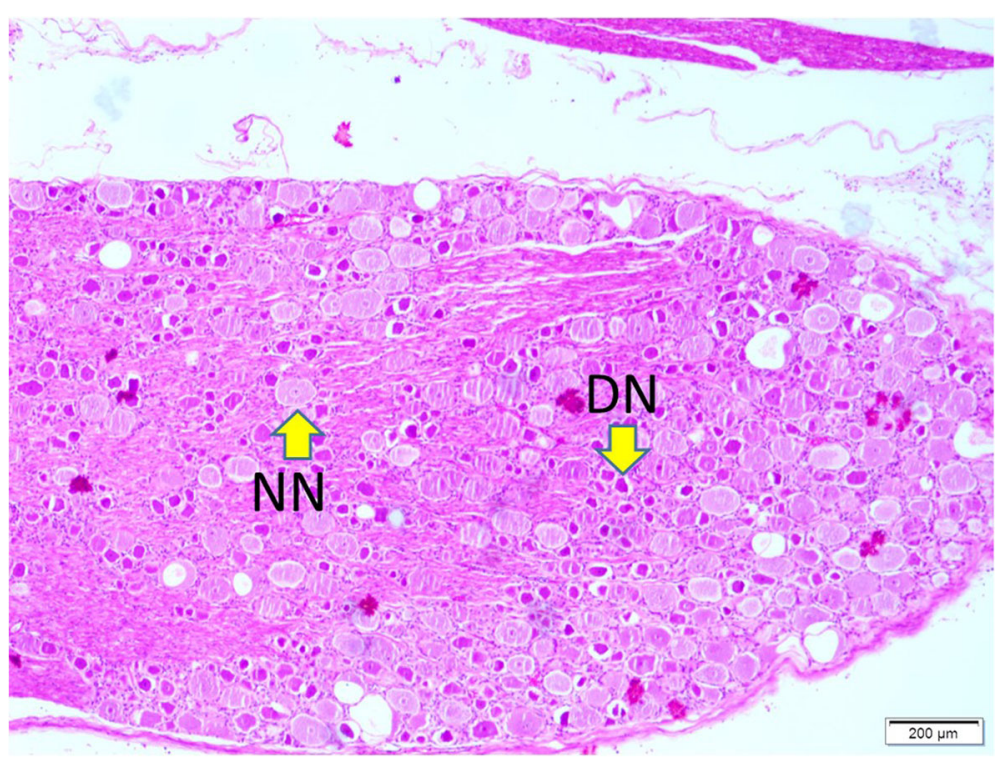

Fig. 8 Histopathological appearance of normal (NN) and degenerated (DN) neurons, including DRG, in an animal that underwent monopolar cautery $(L M, H \& E, \times 10 / A)$

deep and dorsal penile arteries at the ischiatic arch level. The deep penile artery passes through the tunica albuginea and forms the arterial web of corpus cavernosum penis; on the other hand, the dorsal penile artery is divided into three small branches between the subischiocavernosus muscles, while two small branches form an arterial network around the preputium, the dorsolateral surface of the penis and the glans. Moreover, the caudal branch of the prostatic artery sends some branches to penile circulation [2]. During circumcision surgery, the dissected nerves, arteries and veins are mostly pudendal nerves and dorsal penile arteries; therefore, we examined the pudendal nerve ganglia at the bilateral third sacral root ganglia level.

Simple surgical circumcision is still the most commonly used method in male children worldwide [13].

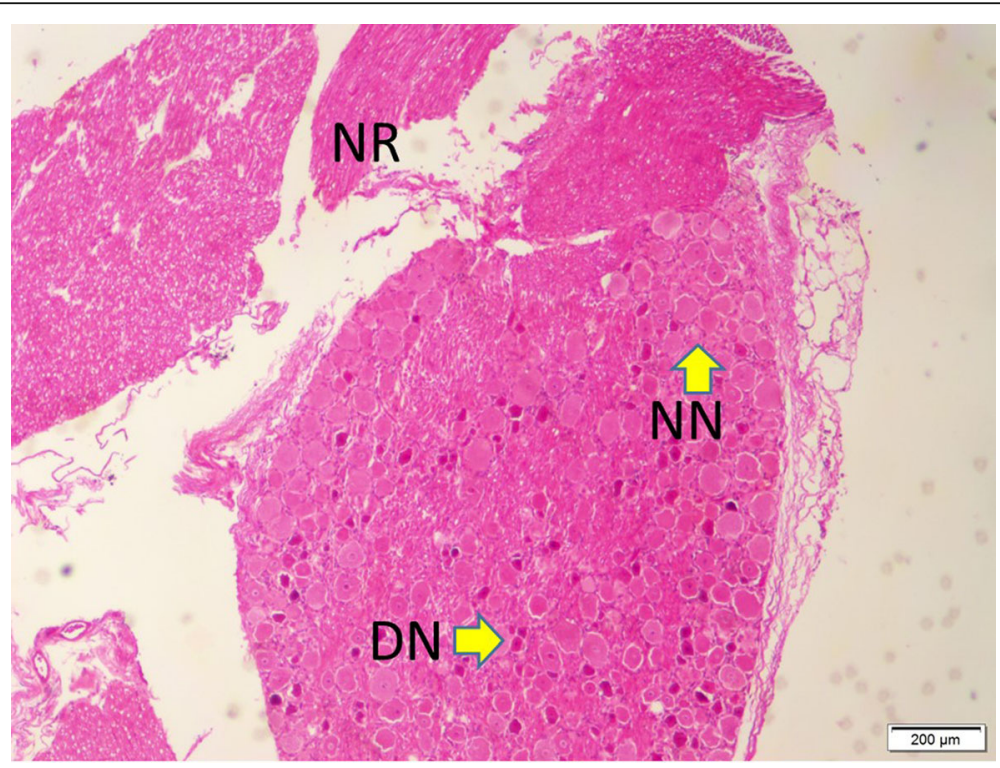

Fig. 9 Histopathological appearance of nerve roots (NR) and normal (NN) and degenerated (DN) neurons, including DRG, in an animal that underwent bipolar cautery $(L M, H \& E, \times 4 / A)$ 
Therefore, circumcision during infancy merely for the nonretractile prepuce is unwarranted, which is the cornerstone for the modern anticircumcision movements [1]. Circumcision is advised for the treatment of phimosis or balanitis [14], although electrocautery damage can be prevented via the tunica albuginea of intracavernous sinusoid insult, which plays an essential role in fulfilling the venoocclusive mechanism [6].

The basic procedure of circumcision is to remove the preputium without causing complications. Although the essential circumcision technique is surgical circumcision, some surgeons use monopolar cautery or thermocautery ablation. Nevertheless, monopolar-bipolar electrocautery devices are also used for postoperative bleeding control. Using bipolar scissors is a safe operation method [15]. Recently, using the Colorado MicroDissection Needle is recommended for easy tissue dissection and separation without complications [16].

Electrical accidents can lead to SC injury complicated by motor, sensory and autonomic dysfunctions. The direct current and heating effect of electric devices can result in neurovascular and SC damage [17].

In circumcision surgery, postoperative bleeding complications occur frequently [5]. If bleeding is potentially dangerous, then electric devices should be used; however, they frequently result in skin bridges and neurovascular complications [18]. Postcircumcision electrocautery penile ablation injuries could lead to vascular injury-related ischaemic insults and scar formation [19]; such pathologies would be more frequent if electric devices are used. Gangrene of the glans penis is the most dangerous complication of electrocautery following circumcision [20]. It is also well-known that electrocauterisation may result in the development of thrombosis [11] and neuronal degeneration in DRG [12]. According to Peng et al., using the Ultracision Harmonic Scalpel in circumcision is a safe method because of its simplicity and lower complication rates [21]. To avoid the hazardous electric current in bipolar scissors, circumcision is advised, since it is more effective and safer than the standard scalpel techniques in terms of morbidity [22]. Electrocautery is more commonly used in separating the prepuce from the glans in newborn circumcision; however, the glans is superficially injured and if the shaft skin or part of the circumcision wound adheres to this, it may cause epithelialisation and growth, causing a skin bridge [23, 24]. The authors recommended that high-voltage electrocautery should not be employed in such circumstances. Since there is no retrograde injury in cautery in bipolar, the damage is less. The same mechanism is also valid for thermocoutery [25]. We think this is the reason why we suffer more damage in monopolar cautery and less damage in others.
Both the literature and our research suggest that the circumcision process should be further investigated in future studies.

\section{Clinical implications of the study}

Sensory innervation of the penile tissues affects wound healing through the immunoregulatory and circulation modulation of nerve endings [26]. Wound healing could be challenging in the presence of neural injuries. Although circumcision may be useful in preventing harboured uropathogenic flora infections [27], using electrical devices induces neural circuitry pathologies such as nonhealing wound and circumcision area infections. Moreover, we demonstrated that electrical device usage might cause penile tissue innervating somatosensitive nerves and their ganglia injury. The following complications should be attributed to neural network collapse following circumcision: penile adhesion, skin bridges, meatal stenosis, redundant foreskin, recurrent phimosis, buried penis and penile rotation [28], residual foreskin, meatal stenosis, granuloma [29] and postcircumcision necrosis [30]. According to our clinical experiences, the granuloma is frequently developed from cauterised nerve endings [12]. For that reason, high-voltage electrical devices should not be used unless necessary.

\section{Limitations}

This was an animal study; as a result, its findings may not be generalisable to humans. If we used local anaesthetics some resembling complications like electrocautery could be possible. Lack of sham group may be another limitation; however, the surgical circumcision group may be considered a sham group. Surgical circumcision methods were compared in this study, but Gomco, plastibell and clamp methods were not included in the study, which is one of the limitations of our study. If the vascular injury is mild, satellite neural and endothelial cells can be regenerated with neovascularisation and collaterals to develop. If apoptotic neurodegeneration has begun, the neurodegeneration is irreversible. However, since this study was short, no evaluation was made regarding whether neuronal degeneration was reversible or irreversible, which is one of the limitations of our study.

\section{Conclusion}

Both electrical and thermal devices can be hazardous to the finely organised neurovascular web of the penile tissue; therefore, it should be used with low voltage current and not be applied unless obligatory. In addition, we think that longer-term studies are needed to understand the late effects of neuronal damage more clearly. 


\section{Future insight}

Although only the pudendal nerve endings secondary to preputium excision are affected, another pudendal axon can be damaged during bleeding control with cautery; the destruction of the neuron sending this axon initiates a general inflammation in that ganglion. As a result, there may be a somatosensitive innervation defect in extrapenile tissues innervated by this ganglion. Moreover, neighbouring ganglia could also be affected, as in our study. For this reason, we concluded that circumcision techniques could cause extrapenile tissue pathologies in the future.

\section{Abbreviations}

DRG: Dorsal root ganglion; DN: Degenerated neuron; NR: Nerve roots; SC: Sacral spinal cord; AKA: Adamkiewicz artery

\section{Acknowledgements}

The authors gratefully acknowledge the financial support by Ataturk University BAP Project grant number 2019/7014.

\section{Authors' contributions}

OC and MDA conceived the study and participated in its design. OC, BF, MEA and KAN contributed to the experimental part of the study. RA made histolpathological examinations. OC, RA and EK participated in the acquisition, analysis, and interpretation of the data. MDA and ABS performed the critical revision. All authors have read and approved the manuscript.

\section{Funding}

Financial support by Ataturk University BAP Department.

\section{Availability of data and materials}

The datasets used and/or analysed during the current study are available from the corresponding author on reasonable request.

\section{Ethics approval and consent to participate}

Ethical approval was obtained from Atatürk University Ethics Committee (ethical number, 17.09.2018/10).

\section{Consent for publication}

No consent for publication was not required for this experimental study.

\section{Competing interests}

The authors declare no competing interests.

\section{Author details \\ ${ }^{1}$ Department of Pediatric Surgery, Medical Faculty, Ataturk University, Erzurum, Turkey. ${ }^{2}$ Department of Pathology, Medical Faculty, Ataturk University, Erzurum, Turkey. ${ }^{3}$ Department of Anesthesiology and Reanimation, Medical Faculty, Ataturk University, Erzurum, Turkey. ${ }^{4}$ Department of General Surgery, Medical Faculty, Ataturk University, Erzurum, Turkey. ${ }^{5}$ Department of Pharmacology, Medical Faculty, Ataturk University, Erzurum, Turkey. ${ }^{6}$ Department of Neurosurgery, Medical Faculty, Ataturk University, Erzurum, Turkey.}

Received: 24 November 2020 Accepted: 4 February 2021 Published online: 07 April 2021

\section{References}

1. Raveenthiran $V$. The evolutionary saga of circumcision from a religious perspective. J Pediatr Surg. 2018;53(7):1440-3.

2. Ozgel $\mathrm{O}$, et al. Arterial supply of the penis in the New Zealand rabbit (Oryctolagus cuniculus L.). Anat Histol Embryol. 2003;32(1):6-8.

3. Aydın MD, et al. Taste bud-like structures in penile tissues and a predictive neural mechanism of male orgasm: a preliminary hypothesis based on histological evidence. Neurol Psychiatry Brain Res. 2014;20(3):55-62.
4. Aydin MD, et al. The sooth beneath the taste roseas in the urethra and first description of neuro-morpho-chemical mechanism of penile erectile posture in males: an experimental study. Int J Neurosci. 2018;128(7):663-9.

5. Harty NJ, et al. The impact of electrocautery method on post-operative bleeding complications after non-newborn circumcision and revision circumcision. J Pediatr Urol. 2013;9(5):634-7.

6. Hsieh $\mathrm{CH}$, et al. Tunical outer layer plays an essential role in penile venoocclusive mechanism evidenced from electrocautery effects to the corpora cavernosa in defrosted human cadavers. Urology. 2015;86(6):1129-35.

7. Concodora CW, et al. Checklist assessment tool to evaluate suitability and success of neonatal clamp circumcision: a prospective study. J Pediatr Urol. 2016;12(4):235.e1-5.

8. Hunt JL, et al. The pathophysiology of acute electric injuries. J Trauma. 1976; 16(5):335-40.

9. Koshima I, et al. High-voltage electrical injury: electron microscopic findings of injured vessel, nerve, and muscle. Ann Plast Surg. 1991;26(6):587-91.

10. Lee RC. Injury by electrical forces: pathophysiology, manifestations, and therapy. Curr Probl Surg. 1997;34(9):677-764.

11. Aydin MD, et al. Thrombogenetic effect of facet denervation using in disc surgery on spinal radicular arteries: an experimental study. Minim Invasive Neurosurg. 2006;49(6):328-30.

12. Aydin MD, et al. Neurodegenerative effects of monopolar electrocauterization on spinal ganglia in lumbar disc surgery. Acta Neurochir. 2004;146(10):1125-9 discussion 1129.

13. Voznesensky $M$, et al. Pediatric sutureless circumcision: an effective and cost efficient alternative. Can J Urol. 2015:22(5):7995-9.

14. Tucker SC, et al. Circumcision: a refined technique and 5 year review. Ann R Coll Surg Engl. 2001;83(2):121-5.

15. Fraser ID, Tjoe J. Circumcision using bipolar diathermy scissors: a simple, safe and acceptable new technique. Ann R Coll Surg Engl. 2000;82(3):190-1.

16. Liang WQ, et al. A comparative study of surgical outcomes using a Colorado microdissection needle versus standard-size needle electrocautery in one-stage hypospadias repair using a transverse preputial island flap. Minerva Pediatr. 2018;70(1):27-34.

17. Varghese G, Mani MM, Redford JB. Spinal cord injuries following electrical accidents. Paraplegia. 1986;24(3):159-66.

18. Naimer SA, et al. Office management of penile skin bridges with electrocautery. J Am Board Fam Pract. 2002;15(6):485-8.

19. Seleim HM, Elbarbary MM. Major penile injuries as a result of cautery during newborn circumcision. J Pediatr Surg. 2016;51(9):1532-7.

20. Uzun $\mathrm{G}$, et al. Electrocautery-induced gangrene of the glans penis in a child following circumcision. BMJ Case Rep. 2012;2012:bcr-2012-007096. https:// doi.org/10.1136/bcr-2012-007096.

21. Peng $\mathrm{M}$, et al. The ultrasonic harmonic scalpel for circumcision: experimental evaluation using dogs. Asian J Androl. 2013;15(1):93-6.

22. Mendez-Gallart R, et al. Bipolar scissors circumcision is a safe, fast, and bloodless procedure in children. J Pediatr Surg. 2009;44(10):2048-53.

23. Klauber GT, Boyle J. Preputial skin-bridging. Complication of circumcision. Urology. 1974;3(6):722-3

24. Ritchey ML, Bloom DA. Re: Skin bridge--a complication of paediatric circumcision. Br J Urol. 1991;68(3):331.

25. Dagtekin A, et al. Comparison of the effects of different electrocautery applications to peripheral nerves: an experimental study. Acta Neurochir. 2011;153(10):2031-9.

26. Nazir Z, Masood R, Rehman R. Sensory innervation of normal and hypospadiac prepuce: possible implications in hypospadiology. Pediatr Surg Int. 2004;20(8):623-7.

27. Wijesinha SS, et al. Does circumcision alter the periurethral bacterial flora? Pediatr Surg Int. 1998;13(2-3):146-8.

28. Pieretti RV, Goldstein AM, Pieretti-Vanmarcke R. Late complications of newborn circumcision: a common and avoidable problem. Pediatr Surg Int. 2010;26(5):515-8.

29. Yegane RA, et al. Late complications of circumcision in Iran. Pediatr Surg Int. 2006;22(5):442-5.

30. Tasci Al, et al. Management of post-circumcision necrosis of the penis: the medicolegal aspect. Pediatr Surg Int. 2020;36(4):523-8.

\section{Publisher's Note}

Springer Nature remains neutral with regard to jurisdictional claims in published maps and institutional affiliations. 